\title{
Management of Non-steroidal Anti-inflammatory Drug-exacerbated Respiratory Disease
}

\author{
Mark Arnold, ${ }^{1}$ Merin Kuruvilla ${ }^{2}$ and Joshua M Levy ${ }^{1}$ \\ 1. Department of Otolaryngology-Head and Neck Surgery, Emory University School of Medicine, Atlanta, GA, USA; \\ 2. Department of Medicine, Emory University School of Medicine, Atlanta, GA, USA
}

\begin{abstract}
A spirin-exacerbated respiratory disease (AERD) is characterized by the clinical triad of asthma, nasal polyposis and sensitivity to aspirin and other non-steroidal anti-inflammatory drugs. Across the spectrum of chronic rhinosinusitis, patients with AERD are among those with the most severe sinopulmonary inflammation, often refractory to standard treatments. Although AERD has a reputation as a recalcitrant disease, presentation and response to treatment is often along a spectrum. Yet, there have recently been significant advances in the management of both asthma and nasal polyposis, allowing for new treatment options and a shift in standard management algorithms. This article explores the prevalence and workup of AERD and reviews the latest evidence in both medical and surgical management. Furthermore, the results of on-going clinical trials are eagerly awaited. With a growing assortment of treatment options, especially with the addition of biologics to the clinician's armamentarium, it is paramount to individualize therapy alongside a stepwise algorithm for what is considered a difficult-to-treat disease.
\end{abstract}

\section{Keywords}

Aspirin-exacerbated respiratory disease, aspirin sensitivity, aspirin desensitization, endoscopic sinus surgery, chronic rhinosinusitis

Disclosures: Joshua M Levy has served on scientific advisory boards for Regeneron Pharmaceuticals and GlaxoSmithKline. Mark Arnold and Merin Kuruvilla have no financial or non-financial relationships or activities to declare in relation to this article.

Review process: Double-blind peer review.

Compliance with ethics: This study involves a review of the literature and did not involve any studies with human or animal subjects performed by any of the authors.

Data availability: Data sharing is not applicable to this article as no datasets were generated or analysed during the writing of this article.

Authorship: The named authors meet the International Committee of Medical Journal Editors (ICMJE) criteria for authorship of this manuscript, take responsibility for the integrity of the work as a whole, and have given final approval for the version to be published.

Digital features: This article is published with a video abstract to enhance understanding. This video was pee reviewed post-acceptance of the manuscript. To view the video please visit https://www.touchrespiratory.com/ allergy/conference-hub/management-of-non-steroidalanti-inflammatory-drug-exacerbated-respiratory-disease

Access: This article is freely accessible at touchRESPIRATORY.com (c) Touch Medical Media 2021.

Received: 15 June 2021

Accepted: 12 July 2021

Published online: 22 December 2021

Citation: touchREVIEWS in Respiratory \&

Pulmonary Diseases. 2021;6(1):21-5

Corresponding author: Joshua M Levy, Emory University Midtown, 550 Peachtree Rd. NE, Suite 1135, Atlanta, GA 30308, USA. E: joshua.levy2@emory.edu

Support: No funding was received in

the publication of this article.
Aspirin-exacerbated respiratory disease (AERD) is a clinical triad of asthma, nasal polyposis and sensitivity to aspirin and other non-steroidal anti-inflammatory drugs (NSAIDS). These patients have severe sinopulmonary inflammation, often refractory to standard treatments. ${ }^{1}$ However, significant advances in the management of both asthma and nasal polyposis allow for new treatment options and management algorithms. This article aims to review the most recent clinical updates of AERD prevalence, diagnosis and management. Recent clinical studies with relevance to patients with AERD are reviewed.

\section{Prevalence}

For the estimated 2 million patients with AERD living in the United States, AERD represents a significant healthcare burden, costing over US $\$ 4.5$ billion, annually. ${ }^{2}$ It is thought that $8-26 \%$ of patients with nasal polyposis and $7.2 \%$ of patients with asthma have AERD. ${ }^{2,3}$ However, the true prevalence of AERD is unknown, as aspirin challenge is underused. ${ }^{4} \mathrm{~A}$ history of sensitivity to aspirin or NSAIDs is suggestive of AERD, but a lack of such a reaction does not rule it out. It has been shown that $30-40 \%$ of patients with nasal polyps who feel they are tolerant to aspirin actually have a positive aspirin challenge. ${ }^{2}$

\section{Pathophysiology}

The sinopulmonary symptoms seen in AERD are due to recalcitrant type-2 inflammation. The inflammatory profile stems from baseline dysregulated arachidonic acid metabolism, mast cell activation and blood and airway eosinophilia. ${ }^{5}$ Dysfunctional metabolism of arachidonic acid has been found to be the key mediator in AERD pathogenesis. ${ }^{6}$ At baseline, patients with AERD have decreased cyclooxygenase-2 (COX-2) and prostaglandin $E_{2}$, resulting in decreased prostaglandins and increased pro-inflammatory pro-leukotrienes. Therefore, cyclooxygenase-1 (COX-1) inhibitors shunt arachidonic metabolism further towards the pro-leukotriene pathway, causing a characteristic acute increase in inflammatory symptoms, often presenting as respiratory, and occasionally, systemic reactions. The downstream product, urinary leukotriene E4 (ULTE4), is elevated in patients with AERD, and has been shown to elevate further during NSAID-induced reactions, such as aspirin desensitization. ${ }^{6}$ Consequently, uLTE4 has been the focus of significant research as a potential biomarker of AERD diagnosis and severity, although it remains undetermined. Importantly, patients with AERD tolerate COX-2 inhibitors. ${ }^{8}$

\section{Diagnosis}

AERD is usually diagnosed in the third or fourth decade of life. ${ }^{9}$ The natural history involves chronic rhinosinusitis that evolves into manifestations of lower airway inflammation, and subsequently, NSAID hypersensitivity. Upper airway symptoms include nasal congestion, facial pressure/pain, rhinorrhoea, loss of smell and sneezing, while lower airway symptoms include laryngospasm, coughing, wheezing and shortness of breath. ${ }^{1}$ As a systemic disease, patients may experience 
gastrointestinal manifestations, primarily abdominal pain, and cutaneous symptoms, such as flushing and urticaria. ${ }^{1}$ These symptoms can develop independently, and are characteristically exacerbated by aspirin and other NSAIDs. The presentation with these symptoms alone is often insufficient for diagnosis, and aspirin challenge may be necessary for confirmation. Interestingly, upper airway symptoms, including rhinitis, often precede the lower airway symptoms of asthma by $1-5$ years. ${ }^{10}$ The chronology of symptoms seems to mimic an 'allergic march', seen in atopic disease, although AERD does not represent a true allergy. Although patients may present with anaphylaxis-like attacks, symptoms of AERD are not immunoglobulin E (IgE) mediated." However, environmental allergies and AERD may co-exist. ${ }^{12}$

The diagnosis of AERD may be difficult, as up to $15 \%$ of patients with AERD are unable to identify if they are sensitive to NSAIDS. ${ }^{9}$ The reasons for this are multifactorial, and include recommended NSAID avoidance following the development of asthma and nasal polyps. Occasionally, patients may even tolerate low-dose aspirin therapy. ${ }^{13}$

The aspirin provocation challenge, performed in a monitored setting, is the gold standard for the diagnosis of AERD. A challenge is considered positive in the event of naso-ocular and/or lower respiratory reactions, and if forced expiratory volume in 1 second $\left(F_{E V}\right)$ decreases by $>15 \%$. The aspirin challenge is safe when preceded by a pre-treatment strategy, including oral corticosteroids and leukotriene-modifying drugs, but should not be offered if the baseline $\mathrm{FEV}_{1}$ is $<70 \%$, or if there is a history of a severe reaction or anaphylaxis to aspirin or other NSAIDs. While it is an integral part of AERD diagnosis, unfortunately the aspirin challenge is underused. ${ }^{4}$ In a survey of physicians by the American Academy of Allergy, Asthma and Immunology (AAAAl), aspirin challenge was not offered by $37.5 \%$ of respondents, and $28.2 \%$ did not refer patients to a provider who would perform the challenge. ${ }^{14}$ Consequently, there exists an undiagnosed and therefore undertreated population with AERD.

Beyond sensitivity to aspirin, other clues in a patient's history may suggest AERD. Smoking cessation has been found to be a risk factor for AERD. ${ }^{15}$ Of 114 patients with AERD, Hayashi et al. found that, with the onset of symptoms, $20 \%$ had recently quit smoking and were 4.63 times more likely to have recently quit compared with patients with aspirin-tolerant asthma. ${ }^{15}$ The underlying mechanism is likely the pro-inflammatory environment caused by smoking, which increases COX-2 expression. In addition, up to $75 \%$ of patients with AERD also display sensitivity to alcohol, with flushing, wheezing and urticaria. ${ }^{16}$ The mechanism is largely unknown, but may be related to polyphenols present in alcohol, which are known inhibitors of COX-1. ${ }^{17}$ However, this phenomenon is observed less frequently in patients with aspirin-tolerant asthma, allergic rhinitis and even in healthy controls as compared to AERD. Importantly, patients often tolerate alcohol after aspirin desensitization or dupilumab therapy. ${ }^{18,19}$ This unique aspect of therapy may be a motivating factor for some patients to undergo these treatments.

\section{Treatment}

\section{Avoidance}

As AERD is exacerbated by aspirin or NSAID ingestion, it seems appropriate that salicylate avoidance would appropriately control symptoms. However, baseline symptoms of AERD tend to be progressive, even with strict NSAID avoidance, as COX-1 inhibition is not necessary to either initiate or sustain the disease. While additional study is required, exposure to dietary non-acetylated salicylates may contribute to this baseline inflammation. ${ }^{22}$ High-salicylate beverages include alcohol, carbonated drinks and coffee; high-salicylate foods include starches, leafy greens and most fruits. In 2015, Sommer et al. performed a prospective, cross-over, pilot study in 10 patients randomized to 6 weeks of either a regular diet or a low-salicylate diet. Results showed improved nasal symptoms and endoscopy scores in the patients on a lowsalicylate diet. ${ }^{20} \mathrm{~A}$ follow-up prospective, cross-over study was recently performed, which randomized seven patients to either a high- or lowsalicylate diet for 1 week. While there was no statistical difference in the urinary salicylate and leukotriene levels between the two diets, patients on the low-salicylate diet had improved sino-nasal outcomes test (SNOT22) scores. ${ }^{21}$ Given the fact that low-salicylate diets are highly restrictive, further research is needed to support these findings..$^{22}$ In addition, dietary omega-3 and omega-6 fatty-acid intake may play a role in disease control by targeting arachidonic acid dysregulation. A prospective, non-blinded study of 10 patients with AERD found a diet reduced in omega- 6 fattyacid consumption with increased omega-3 intake led to reductions in both uLTE4 and SNOT-22 scores, compared with baseline. ${ }^{23}$

\section{Topical therapy}

As an inflammatory process of the upper and lower airways, topical corticosteroid treatment is standard therapy for both the asthma and nasal polyps seen in AERD. While the use of multiple inhalers (including short- and long-acting $\beta$ agonists) may be sufficient to manage lower airway disease, patients with asthma and comorbid AERD are significantly more likely to be oral-corticosteroid dependent than patients with chronic rhinosinusitis with nasal polyposis (CRSWNP) and asthma. ${ }^{24}$ Similarly, topical corticosteroid sprays in the nasal cavity are less successful in controlling upper airway symptoms in patients with asthma and AERD than in patients with CRSWNP; ${ }^{25}$ and unoperated sinuses prevent deep penetration of topical steroid sprays. Furthermore, patients with AERD often have significant nasal polyp burden, resulting in poor corticosteroid distribution. High-volume rinses with off-label corticosteroids, such as budesonide or mometasone, may improve medication delivery. The recent development of novel steroid delivery devices, such as Xhance ${ }^{\circledR}$ (OptiNose US, Yardley, PA, USA), has shown promise in patients with nasal polyposis. ${ }^{26}$ As AERD is often recalcitrant, these delivery devices may be an alternative after exhausting other medical options.

\section{Oral therapies}

A hallmark of AERD is cysteinyl leukotriene overproduction, which is elevated further during acute inflammatory events brought on by aspirin or NSAIDS. ${ }^{27}$ Pro-inflammatory leukotrienes underpin the pathogenesis of AERD; therefore, leukotriene-modifying drugs are a viable option. Montelukast and zafirlukast work as cysteinyl leukotriene receptor 1 (CySLT1) antagonists and are an integral component of aspirin desensitization safety. ${ }^{28}$ While they are often included in the medical regimen of patients with AERD, their benefits in controlling both upper and lower airway symptoms are less clear. Similarly, zileuton acts as a partial inhibitor of 5-lipoxygenase, halting downstream leukotriene formation. 29,30 A recent review of 45 patients with AERD found that those taking zileuton had slightly reduced rates of surgical intervention (though not statistically significant) compared with those not taking the medication; however, no benefit was seen in other rhinologic quality-of-life outcomes. ${ }^{29}$ The side effects of these drugs must also be considered. Montelukast carries a risk of mental health side effects, including completed suicide. ${ }^{31}$ Zileuton carries a risk of self-limited hepatotoxicity, which requires liver function monitoring. ${ }^{32}$

As with other forms of asthma and chronic rhinosinusitis, judicious use of oral steroid therapies provides short-term benefits in patients with AERD. Oral steroids are often best employed in the perioperative period to reduce polyp regrowth after functional endoscopic sinus surgery. ${ }^{22}$ 


\section{Aspirin desensitization}

Aspirin desensitization therapy was discovered unintentionally at the Scripps Clinic (San Diego, CA, USA) in the late 1970s during aspirin challenge clinical trials. ${ }^{33}$ Aspirin desensitization is not directly analogous to other inhalant/medication allergen desensitization, as the underlying mechanism of aspirin desensitization remains unknown. Despite this, there have been several double-blind, placebo-controlled studies demonstrating the benefits of aspirin desensitization. ${ }^{34}$ Principally, longterm, high-dose aspirin therapy affords significant improvement in upper airway symptoms, decreased polyp recurrence, fewer revision surgeries and improved quality-of-life metrics as compared to placebo. ${ }^{34-36}$ However, through all these trials, it should be noted that desensitization is difficult to blind, as symptoms will characteristically develop with aspirin ingestion.

Historically, patients were given incremental doses of aspirin until $650 \mathrm{mg}$ twice daily was tolerated. However, multiple trials have demonstrated a benefit at lower doses. In a trial of 40 patients, as low as $300 \mathrm{mg}$ per day was found to be effective. ${ }^{36}$ Although there was no comparison group, the investigators found favourable outcomes, including fewer episodes of sinusitis and revision surgeries, and improved nasal congestion and sense of smell. ${ }^{36}$ Furthermore, a study performed in 2013 compared just $100 \mathrm{mg} /$ day aspirin against placebo. ${ }^{35}$ With 70 initial participants, 31 individuals were evaluated at 36 months. The authors reported that the aspirin group had reduced nasal polyp relapse. It should be noted, however, that a major limitation of this study was the high dropout rate and patients with AERD were selected based on history and in vitro testing. A recent work-group report recommends the maintenance dose of aspirin start at $650 \mathrm{mg}$ twice daily for 1-6 months, with step down thereafter to $325 \mathrm{mg}$ once or twice daily for patients who are doing well. If symptoms worsen following the step down, the dose should be increased back to $650 \mathrm{mg}$ twice daily.

Aspirin challenges and desensitization are typically performed in an outpatient setting, although prior to the adoption of pre-medication protocols this was frequently completed in the intensive care unit. Aspirin challenges are performed to confirm underlying AERD, whereas aspirin desensitization is indicated for patients who need long-term, high-dose aspirin therapy. The protocol used for both procedures is identical, and both aspirin challenges and desensitization involve invoking symptoms of AERD. Characteristic upper and lower airway symptoms occur within 40-60 minutes. To minimize these reactions, most aspirin challenges move immediately to desensitization. Ocular and gastrointestinal symptoms may also develop. ${ }^{38}$

Most protocols are complete by 24-48 hours of therapy, and several different protocols exist. ${ }^{39,40}$ Patients should be monitored for at least 3 hours following an aspirin-induced reaction during challenges and desensitization until symptom resolution. The protocol is stopped thereafter in a challenge, whereas in a desensitization, the provoking dose is re-administered and the protocol continued through to $325 \mathrm{mg}$ of aspirin. It is important to note that not all protocols use oral aspirin for desensitization. Many European countries use ketorolac or other NSAIDS, as well as intranasal administration. Desensitization continues for at least 6 months, and if effective, indefinitely. ${ }^{39}$ If side effects arise, aspirin therapy should be discontinued at any point. In addition, it should be noted that the desensitization itself is not beneficial, but rather the daily maintenance dose of aspirin following desensitization is what produces the therapeutic effect. ${ }^{35}$

Prior to desensitization, patients are typically pre-medicated with leukotriene inhibitors, with oral-corticosteroids being reserved for
Table 1: Oral aspirin challenge protocol for aspirin desensitization

\begin{tabular}{|l|l|l|}
\hline Time & Day 1 & Day 2 \\
\hline $8 \mathrm{am}$ & $20-40 \mathrm{mg}^{\star}$ & $100-160 \mathrm{mg}$ \\
\hline $11 \mathrm{am}$ & $40-60 \mathrm{mg}$ & $160-325 \mathrm{mg}$ \\
\hline $2 \mathrm{pm}$ & $60-100 \mathrm{mg}$ & $325 \mathrm{mg}$ \\
\hline
\end{tabular}

This is one of many published protocols for aspirin desensitization. Lower

starting doses of aspirin are provided for patients who are not pre-treated with a leukotriene-modifying drug or have a low $\mathrm{FEV}_{1}$. Otherwise, a higher starting dose can be chosen.

- Confirm that the patient's baseline $\mathrm{FEV}_{1}$ is the same as the prior best value, and that they have not used their albuterol rescue inhaler in the past week. If not, consider a 1-day placebo challenge to determine stability of airways.

- *Choice of dosing: a lower dose should be chosen if the patient is not using a leukotriene-modifying drug, has a low baseline $\mathrm{FEV}_{1}$ and has had a recent hospitalization or emergency department visit for asthma.

- Using a pill cutter, an 81-mg ASA tablet can be cut into one-half or onequarter.

- Measure FEV , every hour and wait 3 hours between doses.

- $\mathrm{FEV}_{1}$ should be $\geq 1.5 \mathrm{~L}$ and $>60 \%$ of predicted value.

- After a reaction has been treated and resolved, go to step A:

A. Repeat the ASA-provoking dose.

B. If no reaction, continue to escalate the dose every 3 hours as above.

C. At $325 \mathrm{mg}$ of ASA, desensitization/tolerance is complete.

D. The patient should be instructed to start $650 \mathrm{mg}$ of ASA that night as their first dose and continue with $650 \mathrm{mg}$ twice daily.

ASA = acetylsalicylic acid; $F E V_{1}=$ forced expiratory volume in 1 second Reprinted from Journal of Allergy and Clinical Immunology, 133, Katharine M. Woessner and Andrew A. White, Evidence-based approach to aspirin desensitization in aspirinexacerbated respiratory disease, 286-7.e1-9, 2014, with permission from Elsevier. ${ }^{39}$

patients with tenuous asthma control. A full 'crash-cart' or airway box is required, stocked with appropriate medications including epinephrine and antihistamines. Patients undergoing desensitization may continue their biologics and inhaled corticosteroids. Incentive spirometry is used to monitor $\mathrm{FEV}_{1}$ closely. ${ }^{41} \mathrm{~A}$ widely used protocol from Scripps Clinic starts the first day with 20-40 mg of aspirin, doubling the dose every 3 hours for 6 hours. ${ }^{39}$ The following day, the dose starts at 100-160 mg of aspirin and increases every 3 hours until $325 \mathrm{mg}$ is tolerated. That night, the patient is desensitized and takes $650 \mathrm{mg}$ of aspirin. FEV ${ }_{1}$ should be checked every hour and remain $>1.5 \mathrm{~L}$ or $>60 \%$ of the predicted value. With this protocol, patients are desensitized over 2 days during office hours. This protocol is summarized in Table $1.39 \mathrm{It}$ should be noted that there are recent published trials demonstrating success in just 1 day of the oral aspirin challenge. ${ }^{42}$ Furthermore, intranasal ketorolac challenge and desensitization followed by rapid oral aspirin challenge is also safe and less time-consuming than the standard oral aspirin desensitization protocol. ${ }^{43}$

While aspirin desensitization is generally safe, there are contraindications. Patients who are pregnant, have unstable asthma, gastric ulcers, eosinophilic oesophagitis or are taking other anticoagulants are not candidates for chronic aspirin therapy. In addition, patients under the age of 14 years should not take aspirin due to the risk of Reye's syndrome. ${ }^{37}$

\section{Surgical management}

As a difficult-to-treat variant of chronic rhinosinusitis with nasal polyposis, surgical management of AERD with endoscopic sinus surgery is routine after the failure of appropriate medical therapy. In patients with AERD, endoscopic sinus surgery generally includes the opening of all sinuses. Despite this, the failure rate is high, and many undergo revision, with rates ranging from $38-89 \% .{ }^{44,45}$ The literature generally agrees that 'full-house' endoscopic sinus surgery, or the 
opening of all the paranasal sinuses, is indicated for patients with AERD, though the extent of frontal sinus surgery remains controversial. Some advocate for an upfront Lothrop or Draf III procedure, whereby the bilateral frontal sinuses are made into a common cavity by removing portions of the intersinus septum and middle turbinate attachments. ${ }^{46}$ In a prospective study of 31 patients with AERD who underwent a Lothrop procedure, in addition to standard endoscopic sinus surgery, a failure rate of $22.5 \%$ was reported, whereby patients required revision surgery. ${ }^{47}$ While upfront, maximum-extent, sinus surgery with a Lothrop procedure is an option, the failure rate remains high, highlighting the need for adjunctive therapy. Therefore, the best outcomes occur with strong multidisciplinary care between the surgeon and allergy/ immunology colleagues.

Given the well-studied benefits of aspirin desensitization, this therapy is commonly started 2 weeks after surgery to minimize the bleeding risk. The complementary nature of surgery with long-term, high-dose aspirin therapy was evidenced by Jerschow et al. in $2019 .{ }^{48} \mathrm{~A}$ total of 28 patients underwent aspirin challenge before endoscopic sinus surgery to confirm a diagnosis of AERD. Next, 2-3 weeks after surgery, these patients underwent aspirin challenge followed by aspirin desensitization. Aspirin challenge following endoscopic sinus surgery resulted in less-severe reactions in all patients, and $43 \%$ of patients had no detectable reaction to aspirin. This suggests that decreasing the polyp load decreases the systemic reaction to aspirin. Furthermore, the reduced severity of reaction to aspirin was linked to certain biomarkers, as these patients had increased lipoxin A4, and decreased ULTE4 and prostaglandin D2. Overall, their prospective observational trial demonstrated that aspirin challenge was safer after endoscopic sinus surgery. ${ }^{48}$

\section{Medical management: biologics}

Despite appropriate medical and surgical management, many patients with AERD remain symptomatic. Some may also not derive benefit from, nor tolerate chronic aspirin therapy. Biologic therapy targeting Th2 inflammatory pathways has recently become a therapeutic alternative. Dupilumab, omalizumab and mepolizumab are options for use in moderate-to-severe asthma, and have been studied in patients with AERD. ${ }^{49}$

Dupilumab, approved by the US Food and Drug Administration (FDA) in June 2019 for nasal polyps, is an antagonist of the interleukin-4 receptor (IL-4R) $\alpha$ subunit, modulating both the IL-4 and IL-13 pathways. ${ }^{50}$ Prior studies have shown the effectiveness of dupilumab in treating patients with CRSWNP, ${ }^{51}$ with parallel reduction in local Th2 pro-inflammatory biomarkers on nasal secretion analysis. ${ }^{52}$ Furthermore, in a nested analysis of the phase II dupilumab trial, patients with CRSWNP and underlying AERD (19 patients of 60) demonstrated greater improvements in nasal polyp and SNOT-22 scores compared with the rest of the cohort. ${ }^{53}$ In 2020, Mustafa et al. followed 10 patients with AERD treated with placebo for 1 month, followed by dupilumab as add-on therapy for 6 months. ${ }^{19}$ In addition to improved SNOT-22 and Lund-MacKay computed tomography scores, most patients also had improved tolerance to alcohol, a result which was reproduced in a separate case series..$^{54}$

While omalizumab (anti-IgE) has been approved by the FDA for asthma and idiopathic urticaria since 2003, in December 2020, it joined dupilumab

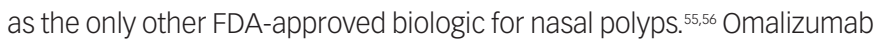
is effective in reducing allergic inflammation by binding circulating IgE. It has been found to be effective in relieving nasal symptoms of patients with AERD, but currently, there is no evidence that it prevents polyp recurrence after surgery. ${ }^{57}$ In a case series of 29 patients, omalizumab reduced the number of oral steroid courses along with short-acting $\beta$ agonist use. ${ }^{58}$ Following this, a randomized, placebo-controlled, double-blind, crossover-trial of 16 patients with AERD, by Hayashi et al. in 2020, demonstrated decreased ULTE4 and reduced upper and lower respiratory tract symptoms after 3 months of omalizumab during oral aspirin challenge. ${ }^{59}$ However, in two phase III studies of omalizumab in patients with nasal polyps, 40 of 265 patients had underlying AERD, and demonstrated similar mean improvements in nasal polyp score at week 24 compared to patients without AERD. ${ }^{60}$

Mepolizumab binds to and inhibits IL-5, preventing the binding with and activation of eosinophils. While a small trial of 20 patients with nasal polyps showed a reduction in polyp size in 12 patients, it remains unclear if mepolizumab provides any specific additional benefit in AERD. ${ }^{49,61}$ Mepolizumab was specifically evaluated in a small retrospective study of 14 patients with AERD, and there was a consistent trend towards decreased SNOT-22 scores, improved sense of smell and improved nasal congestion scores, compared with baseline. ${ }^{62} \mathrm{~A}$ subgroup analysis of the recent phase III study of mepolizumab in patients with nasal polyps also suggested higher efficacy compared with placebo in patients with AERD, based on greater improvement in visual analogue scale score in this population. ${ }^{63}$

With promising early data, future studies of biologics in the treatment of AERD will bring more evidence to the decision-making process. While there are some data supporting the efficacy of omalizumab and mepolizumab, dupilumab currently carries the most evidence of success in treating patients with AERD. 19,53,51 Future head-to-head trials are eagerly awaited. In addition, there are several other biologics in the pipeline, directed against various signalling molecules in the type 2 inflammatory cascade, such as cytokine thymic stromal lymphopoietin (TSLP), which is a significant contributor to the pathophysiology of AERD. ${ }^{64}$ However, access and cost remain significant barriers to their widescale use. Biologics cost $\$ 30,000-40,000 /$ year, with variable cost to payors and patients. ${ }^{25}$ In addition, studies have yet to examine the specific role of biologics after endoscopic sinus surgery; surgery was considered an outcome measure in the aforementioned trials. ${ }^{51,52,61}$ Certainly, with the known benefit of aspirin desensitization after endoscopic sinus surgery, the additional role for biologics in this setting remains unclear.

\section{Management strategy}

Although AERD has a reputation as a recalcitrant disease, presentation and response to treatment is often along a spectrum. With a growing assortment of treatment options, it is paramount to individualize therapy alongside a stepwise algorithm. Patients with AERD should begin with appropriate medical management, including saline irrigations, topical nasal corticosteroids, anti-leukotrienes and the occasional course of oral steroids. ${ }^{25}$ For those that fail, comprehensive sinus surgery is indicated. For those that relapse after a first surgery, a revision with a Lothrop procedure is indicated, unless the first surgery was felt to be inadequate. ${ }^{47}$ Either with early polyp recurrence or, if indicated, following the second endoscopic sinus surgery, adjunctive medical therapies, such as aspirin desensitization, are essential. Patients with polyp recurrence after surgery may be candidates for revision surgery, and removal of these polyps facilitates safe aspirin desensitization and prevents relapse. If aspirin desensitization fails, or is otherwise unable to be tolerated, biologics are an appropriate alternative. ${ }^{48,51}$ 
1. White $A A$, Stevenson DD. Aspirin-exacerbated respiratory disease. N Eng/ I Med. 2018;379:1060-70.

2. Nabavi M, Esmaeilzadeh $\mathrm{H}$, Arshi S, et al. Aspirin hypersensitivity in patients with chronic rhinosinusitis and nasal polyposis: frequency and contributing factors. Am I Rhinol Allergy. 2014:28:239-43.

3. Rajan JP, Wineinger NE, Stevenson DD, White AA. Prevalence of aspirin-exacerbated respiratory disease among asthmatic patients: a meta-analysis of the literature. J Allergy Clin Immunol. 2015;135:676-81.e1.

4. Roland LT, Nagy C, Wang H, et al. Treatment practices for aspirin-exacerbated respiratory disease: analysis of a national insurance claims database. Int Forum Allergy Rhinol. 2020;10:190-3.

5. Laidlaw TM, Boyce JA. Aspirin-exacerbated respiratory disease - new prime suspects. N Engl J Med. 2016;374:484-8.

6. Christie PE, Tagari P, Ford-Hutchinson AW, et al. Urinary leukotriene E4 concentrations increase after aspirin challenge in aspirin-sensitive asthmatic subjects. Am Rev Respir Dis. 1991:143:1025-9.

7. Bochenek G, Stachura T, Szafraniec K, et al. Diagnostic accuracy of urinary LTE4 measurement to predict aspirin-exacerbated respiratory disease in patients with asthma. J Allergy Clin Immunol Pract. 2018;6:528-35.

8. Amrol DJ. COX-2 inhibitors are safe in patients with aspirinexacerbated respiratory disease. NEJM Journal Watch. 201 Available at: www.jwatch.org/na35192/2014/07/24/cox-2inhibitors-are-safe-patients-with-aspirin (accessed 12 October 2021)

9. Szczeklik A, Nizankowska E, Duplaga M. Natural history of aspirin-induced asthma. AIANE Investigators. European Network on Aspirin-Induced Asthma. Eur Respir J. 2000;16:432-6.

10. Stevenson DD, Szczeklik A. Clinical and pathologic perspectives on aspirin sensitivity and asthma. J Allergy Clin Immunol. 2006;118:773-86.

11. Roland LT, Wang H, Mehta CC, et al. Longitudinal progression of aspirin-exacerbated respiratory disease: analysis of a national insurance claims database. Int Forum Allergy Rhinol. 2019;9:1420-3.

12. Berges-Gimeno MP, Simon RA, Stevenson DD. The natural history and clinical characteristics of aspirin-exacerbated respiratory disease. Ann Allergy Asthma Immunol. 2002;89:474-8.

13. Lee-Sarwar K, Johns C, Laidlaw TM, Cahill KN. Tolerance of daily low-dose aspirin does not preclude aspirin-exacerbated respiratory disease. J Allergy Clin Immunol Pract. 2015;3:449-51

14. Waldram JD, White AA. A survey of aspirin desensitization practices among allergists and fellows in training in the United States. J Allergy Clin Immunol Pract. 2016;4:1253-5.

15. Hayashi H, Fukutomi Y, Mitsui C, et al. Smoking cessation as a possible risk factor for the development of aspirin-exacerbated respiratory disease in smokers. J Allergy Clin Immunol Pract. respiratory disease

16. Cardet JC, White AA, Barrett NA, et al. Alcohol-induced respiratory symptoms are common in patients with aspirin exacerbated respiratory disease. J Allergy Clin Immunol Pract. 2014;2:208-13.

17. Payne SC. Re: Alcohol-induced respiratory symptoms are common in patients with aspirin exacerbated respiratory disease. J Allergy Clin Immunol Pract. 2014;2:644.

18. Glicksman JT, Parasher AK, Doghramji L, et al. Alcohol-induced respiratory symptoms improve after aspirin desensitization in patients with aspirin-exacerbated respiratory disease. Int Forum Allergy Rhinol. 2018;8:1093-7.

19. Mustafa SS, Vadamalai K, Scott B, Ramsey A. Dupilumab as add-on therapy for chronic rhinosinusitis with nasal polyposis in aspirin exacerbated respiratory disease. Am J Rhinol Allergy. 2021:35:399-407.

20. Sommer DD, Hoffbauer S, Au M, et al. Treatment of aspirin exacerbated respiratory disease with a low salicylate diet: a pilo crossover study. Otolaryngol Head Neck Surg. 2015;152:42-7.

21. Sowerby $\sqcup$, Patel KB, Schmerk C, et al. Effect of low salicylat diet on clinical and inflammatory markers in patients with aspirin exacerbated respiratory disease - a randomized crossover trial. J Otolaryngol Head Neck Surg. 2021;50:27.

22. Levy JM, Rudmik L, Peters AT, et al. Contemporary management of chronic rhinosinusitis with nasal polyposis in aspirinexacerbated respiratory disease: an evidence-based review with recommendations. Int Forum Allergy Rhinol. 2016;6:1273-83.

23. Schneider $T R$, Johns $C B$, Palumbo ML, et al. Dietary fatty acid modification for the treatment of aspirin-exacerbated respiratory disease: a prospective pilot trial. J Allergy Clin Immunol Pract. 2018;6:825-31.

24. Stevens WW, Peters AT, Hirsch AG, et al. Clinical characteristics of patients with chronic rhinosinusitis with nasal polyps, asthma, and aspirin-exacerbated respiratory disease. J Allergy Clin Immunol Pract. 2017;5:1061-70.e3.

25. White AA, Woessner K, Simon R. Aspirin-exacerbated respiratory disease: update on medical management. World $J$ Otorhinolaryngol Head Neck Surg. 2020;6:241-7.

26. Sindwani R, Han JK, Soteres DF, et al. NAVIGATE I: randomized, placebo-controlled, double-blind trial of the exhalation delivery system with fluticasone for chronic rhinosinusitis with nasal polyps. Am J Rhinol Allergy. 2019;33:69-82.

27. Laidlaw TM, Kidder MS, Bhattacharyya N, et al. Cysteinyl leukotriene overproduction in aspirin-exacerbated respiratory disease is driven by platelet-adherent leukocytes. Blood. 2012;119:3790-8

28. White $A$, Ludington $E$, Mehra $P$, et al. Effect of leukotriene modifier drugs on the safety of oral aspirin challenges. Ann Allergy Asthma Immunol. 2006;97:688-93.

29. Lee SE, Farquhar DR, Adams KN, et al. Effect of zileuto treatment on sinonasal quality of life in patients with aspirin-exacerbated respiratory disease. Am J Rhinol Allergy. 2019;33:791-5

30. Rossi A, Pergola C, Koeberle A, et al. The 5-lipoxygenase inhibitor, zileuton, suppresses prostaglandin biosynthesis by inhibition of arachidonic acid release in macrophages. $\mathrm{Br} J$ Pharmacol. 2010;161:555-70

31. US Food \& Drug Administration. FDA requires Boxed Warning about serious mental health side effects for asthma and allergy drug montelukast (Singulair); advises restricting use for allergic rhinitis. 13 March 2020. Available at: www.fda.gov/drugs/ drug-safety-and-availability/fda-requires-boxed-warning-aboutserious-mental-health-side-effects-asthma-and-allergy-drug serious-mental-health-side-e

32. LiverTox: clinical and research information on drug-induced liver injury [Internet]. 2012. Available at: https://pubmed.ncbi.nlm.nih gov/31643176/ (accessed 12 October 2021).

33. Stevenson DD, Simon RA, Mathison DA. Aspirin-sensitive asthma: tolerance to aspirin after positive oral aspir challenges. J Allergy Clin Immunol. 1980;66:82-8.

34. Esmaeilzadeh $\mathrm{H}$, Nabavi M, Aryan Z, et al. Aspirin desensitization for patients with aspirin-exacerbated respiratory disease: a randomized double-blind placebo-controlled trial. Clin Immuno. 2015;160:349-57.

35. Fruth K, Pogorzelski B, Schmidtmann I, et al. Low-dose aspirin desensitization in individuals with aspirin-exacerbated respiratory disease. Allergy. 2013;68:659-65.

36. Comert S, Celebioglu E, Yucel T, et al. Aspirin $300 \mathrm{mg} /$ day is effective for treating aspirin-exacerbated respiratory disease Allergy. 2013;68:1443-51.

37. Stevens WW, Jerschow E, Baptist AP, et al. The role of aspirin desensitization followed by oral aspirin therapy in managing patients with aspirin-exacerbated respiratory disease: a Work Group Report from the Rhinitis, Rhinosinusitis and Ocular Allerg/ Committee of the American Academy of Allergy, Asthma \& Immunology. J Allergy Clin Immunol. 2021;147:827-44.

38. Simon R. Aspirin-exacerbated respiratory disease: NSAID challenge and desensitization. 2021. Available at: www. uptodate com/contents/aspirin-exacerbated-respiratorydisease-nsaid-challenge-and-desensitization (accessed 12 October 2021).

39. Woessner KM, White AA. Evidence-based approach to aspirin desensitization in aspirin-exacerbated respiratory disease. J Allergy Clin Immunol. 2014:133:286-7.e9.

40. Chen JR, Buchmiller BL, Khan DA. An hourly dose-escalation desensitization protocol for aspirin-exacerbated respiratory disease. J Allergy Clin Immunol Pract. 2015;3:926-31.e1.

41. Lee RU, Stevenson DD. Aspirin-exacerbated respiratory disease: evaluation and management. Allergy Asthma Immunol Res. 2011;3:3-10

42. DeGregorio GA, Singer J, Cahill KN, Laidlaw T. A 1-day, 90-minute aspirin challenge and desensitization protocol in aspirinexacerbated respiratory disease. J Allergy Clin Immunol Pract. 2019;7:1174-80.

43. Lee RU, White AA, Ding D, et al. Use of intranasal ketorolac and modified oral aspirin challenge for desensitization of aspirinexacerbated respiratory disease Ann Allergy Asthma Immunol.
2010;105:130-5

44. Amar YG, Frenkiel S, Sobol SE. Outcome analysis of endoscopic sinus surgery for chronic sinusitis in patients having Samter's triad. I Otolaryngol. 2000:29:7-12.

45. Mendelsohn $D$, Jeremic $G$, Wright ED, Rotenberg BW. Revision rates after endoscopic sinus surgery: a recurrence analysis. Ann Otol Rhinol Laryngol. 2011;120:162-6.

46. Muhonen EG, Goshtasbi K, Papagiannopoulos P, Kuan EC. Appropriate extent of surgery for aspirin-exacerbated respiratory disease. World I Otorhinolaryngol Head Neck Surg. 2020;6:235-240.

47. Morrissey DK, Bassiouni A, Psaltis AJ, et al. Outcomes of modified endoscopic Lothrop in aspirin-exacerbated respiratory disease with nasal polyposis. Int Forum Allergy Rhinol. 2016;6:820-5.

48. Jerschow E, Edin ML, Chi Y, et al. Sinus surgery is associated with a decrease in aspirin-induced reaction severity in patients with aspirin exacerbated respiratory disease. J Allergy Clin Immunol Pract. 2019:7:1580-8.

49. Laidlaw TM, Israel E. Aspirin-exacerbated respiratory disease. 2019. Available at: www.uptodate.com/contents/aspirinexacerbated-respiratory-disease (accessed 12 October 2021).

5. US Food \& Drug Administration. Drug Trials Snapshots: DUPIXENT. 7 July 2020. Available at: www.fda.gov/drugs/ drug-approvals-and-databases/drug-trials-snapshots-dupixent (accessed 12 October 2021)

51. Bachert C, Han JK, Desrosiers M, et al. Efficacy and safety of dupilumab in patients with severe chronic rhinosinusitis with nasal polyps (LIBERTY NP SINUS-24 and LIBERTY NP SINUS-52): results from two multicentre, randomised, double-blind, placebo-controlled, parallel-group phase 3 trials. Lancet. 2019;394:1638-50.

52. Jonstam K, Swanson BN, Mannent LP, et al. Dupilumab reduces local type 2 pro-inflammatory biomarkers in chronic rhinosinusitis with nasal polyposis. Allergy. 2019;74:743-52.

53. Laidlaw TM, Mullol J, Fan C, et al. Dupilumab improves nasal polyp burden and asthma control in patients with CRSWNP and AERD. J Allergy Clin Immunol Pract. 2019;7:2462-5.e1.

54. Arnold M, Kuruvilla M, Levy JM, Xu J. Dupilumab improves alcohol tolerance in aspirin exacerbated respiratory disease. Ann Allergy Asthma Immunol. 2021;127:379-81.

55. US Food \& Drug Administration. Xolairø (omalizumab) for injection, for subcutaneous use. Highlights of prescribing information. 2016. Available at: www.accessdata.fda.gov/ drugsatfda_docs/label/2016/103976s5225lbl.pdf (accessed 13 October 2021)

56. US Food \& Drug Administration. FDA approves first treatment for chronic rhinosinusitis with nasal polyps. 2020. Available at: https://www.fda.gov/news-events/press-announcements/ fda-approves-first-treatment-chronic-rhinosinusitis-nasal-polyps (accessed 6 December 2021).

57. Kowalski ML, Agache I, Bavbek S, et al. Diagnosis and management of NSAID-exacerbated respiratory disease (N-ERD) - a EAACI position paper. Allergy. 2019;74:28-39.

58. Jean T, Eng V , Sheikh J, et al. Effect of omalizumab on outcomes in patients with aspirin-exacerbated respiratory disease. Allergy Asthma Proc. 2019;40:316-20.

59. Hayashi H, Fukutomi Y, Mitsui C, et al. Omalizumab for aspirin hypersensitivity and leukotriene overproduction in aspirinexacerbated respiratory disease. A randomized controlled trial. Am J Respir Crit Care Med. 2020;201:1488-98.

60. Gevaert P, Omachi TA, Corren J, et al. Efficacy and safety of omalizumab in nasal polyposis: 2 randomized phase 3 trials. Allergy Clin Immunol. 2020;146:595-605.

61. Gevaert P, Van Bruaene N, Cattaert T, et al. Mepolizumab, a humanized anti-IL-5 mAb, as a treatment option for severe nasal polyposis J Allergy Clin Immunol. 2011:128:989-95-e1-8.

62. Tuttle KL, Buchheit KM, Laidlaw TM, Cahill KN. A retrospective analysis of mepolizumab in subjects with aspirin-exacerbated respiratory disease. J Allergy Clin Immunol Pract. 2018;6:1045-7.

63. Han JK, Bachert C, Fokkens W, et al. Mepolizumab for chron rhinosinusitis with nasal polyps (SYNAPSE): a randomised, double-blind, placebo-controlled, phase 3 trial. Lancet Respir Med. 2021;9:1141-53.

64. Buchheit KM, Cahill KN, Katz HR, et al. Thymic stroma lymphopoietin controls prostaglandin D2 generation in patients with aspirin-exacerbated respiratory disease. J Allergy Clin Immunol. 2016;137:1566-76.e5. 